\title{
Green chemistry approach for the synthesis of gold nanoparticles with gum kondagogu: characterization, catalytic and antibacterial activity
}

\author{
G. Bhagavanth Reddy • A. Madhusudhan • \\ D. Ramakrishna $\cdot$ D. Ayodhya $\cdot$ M. Venkatesham $\cdot$ \\ G. Veerabhadram
}

Received: 11 December 2014/ Accepted: 12 February 2015/Published online: 24 February 2015

(C) The Author(s) 2015. This article is published with open access at Springerlink.com

\begin{abstract}
Gold nanoparticles (AuNPs) were prepared from $\mathrm{HAuCl}_{4}$ using gum kondagogu, by adopting green synthesis, which is a simple, low cost and ecofriendly technique. The gum kondagogu (Cochlospermum gossypium) serves as both reducing agent and stabilizer. The formation of the AuNPs was identified through the change in the color of the solution from yellow to red. The synthesized AuNPs were characterized by various techniques. The green synthesized AuNPs were found to be stable in the $\mathrm{pH}$ range $\mathrm{pH} 2-12$ and up to the concentration of $5 \mathrm{M} \mathrm{NaCl}$. The stabilized AuNPs demonstrated the excellent catalytic activity in reducing $p$-nitrophenol to $p$-aminophenol in the presence of a reducing agent, $\mathrm{NaBH}_{4}$. The effects of catalyst dose and temperature were studied. The synthesized, new gum-based catalyst was very efficient, easy to prepare, stable, cost-effective and ecofriendly. The synthesized AuNPs showed good antibacterial activity.
\end{abstract}

Keywords Gold nanoparticles - Gum kondagogu · Catalytic activity $\cdot$ Antibacterial activity $\cdot$ Nanomaterials

\section{Introduction}

In recent years, nanomaterials play a substantial role in science and technology due to their unique shape, size, peculiar properties and wide range of potential applications. Metal nanoparticles possessing considerable

G. B. Reddy · A. Madhusudhan · D. Ramakrishna

D. Ayodhya $\cdot$ M. Venkatesham $\cdot$ G. Veerabhadram $(\bowtie)$

Department of Chemistry, University College of Science, Osmania University, Hyderabad, Telangana State 500007, India

e-mail: gvbhadram@gmail.com electronic, chemical and optical [1,2] properties, are different from the bulk materials. Among the several metal nanoparticles, AuNPs have attracted the attention of scientists due to their numerous applications in catalysis, sensing, imaging, and diagnostics [3-6]. 'AuNPs' is the area of research interest due to their unique properties such as tunable surface plasmon resonance (SPR), surface-enhanced Raman scattering, electrical, magnetic, thermal conductivity, antibacterial activity, chemical and biostability [7-13]. Besides this, the use of AuNPs as potential materials in the field of drug delivery and DNA delivery systems is noteworthy $[14,15]$. Most of the available methods for the synthesis of AuNPs involve photochemical reduction, chemical reduction, and electrochemical reduction [16-18]. The reagents used in these methods are $\mathrm{NaBH}_{4}$, hydrazine, ascorbic acid and amino acids which are capable of being oxidized. However, the uses of such chemical reagents that are toxic and ecologically injurious are to be avoided in the synthesis of AuNPs [19, 20]. Hence, the above-mentioned chemical methods are nonecofriendly in nature and have inherent drawbacks. The replacement of non-ecofriendly synthesis methods with clean, non-toxic and globally acceptable green chemistry methods [21] is the current need in the synthesis of AuNPs. Several biological systems such as bacteria, fungi, fruit extract and plants can actively reduce metal ions to form metal nanoparticles in an ecofriendly manner [22-24]. Among these, gums obtained from plants such as gum gellan, chitosan and katira gum, (natural polymers), etc., act as reducing agents and stabilizing agents [25-27]. They are also known to be the best and suitable for large-scale green synthesis.

Gum kondagogu is a naturally available polysaccharide component. This gum is basically cheap, easily available, and non-toxic and has a potential application as a food 
additive [28]. It is extracted from the bark of Cochlospermum gossypium (Bixaceae family) and is largely collected by tribes. The primary structure of gum kondagogu is made up of sugars such as galactose, arabinose, mannose, glucose, gluconic acid, rhamnose and galacturonic acid with sugar linkage of $(1 \rightarrow 2) \beta$-D-Gal $p$, $(1 \rightarrow 4) \beta$-D-Glc $p,(1 \rightarrow 6) \beta$-D-Gal $p, 4-O$-Me- $\alpha$-D-Glc $p$, $(1 \rightarrow 2) \alpha$-L-Rha [29]. Gum kondagogu is an acidic gum and carboxylic acid, acetyl, hydroxyl and carbonyl groups are identified as major functional groups in the gum. There were some reports on the synthesis of silver nanoparticles using gum kondagogu as a reducing and stabilizing agent [30].

Gold as a bulk material has usually been regarded to be inactive as a catalyst. But gold in the form of nanoparticles shows excellent catalytic activity towards the reduction of ferrocyanate(III), deoxygenation of epoxides into alkenes, reduction of nitroarenes and cyanosilylation of aldehydes by TMSCNf $[31,32]$. The $p$-nitrophenol (4-NP), as with other nitrophenols and derivatives, is a common by-product from the production of herbicides, pesticides, and synthetic dyes [33]. Nitrophenols (NP) are environmental poisons due to their toxicity and are inhibitory in nature [34]. In addition, nitrophenols have high solubility and stability in water. These NPs tend to get accumulated in deep soil and stay indefinitely. 4-NP poses a hazard to living beings due to their carcinogenic and recalcitrant properties [35]. Due to this reason, the reduction of 4-NP into $p$-amino phenol (4-AP) is prominent. Sodium borohydride $\left(\mathrm{NaBH}_{4}\right)$ is a strong reducing agent. However, it has no ability to reduce the nitrophenol. Hence, $\mathrm{NaBH}_{4}$ is not effective in this reaction except provided with some catalyst to reduce the kinetic barrier of the reaction. A variety of catalysts were used in the past and recently, $\mathrm{Pt}, \mathrm{Ag}[36,37]$ nanoparticles have been used for the same purpose. Antimicrobial agents are important weapons in fighting bacterial infections and have significantly benefited the health-related quality of human life [38]. Organic compounds used as disinfectants have some disadvantages, including toxicity to the human body. For that reason, the interest in inorganic disinfectants such as metal nanoparticles (NPs) is increasing. In addition, AuNPs with smaller particle size have been reported to show good antimicrobial activity [39].

The present study reports the green synthesis of AuNPs using the gum kondagogu as an essential material. This gum serves as both reducing and stabilizing agent. The characterization of AuNPs was carried out by UV-Visible spectrophotometer, FTIR spectroscopy, XRD analysis and TEM. The AuNPs were explored with respect to their prospective catalytic applications. The antibacterial activity of AuNPs was tested against Gram-positive and Gram-negative strains of bacteria.

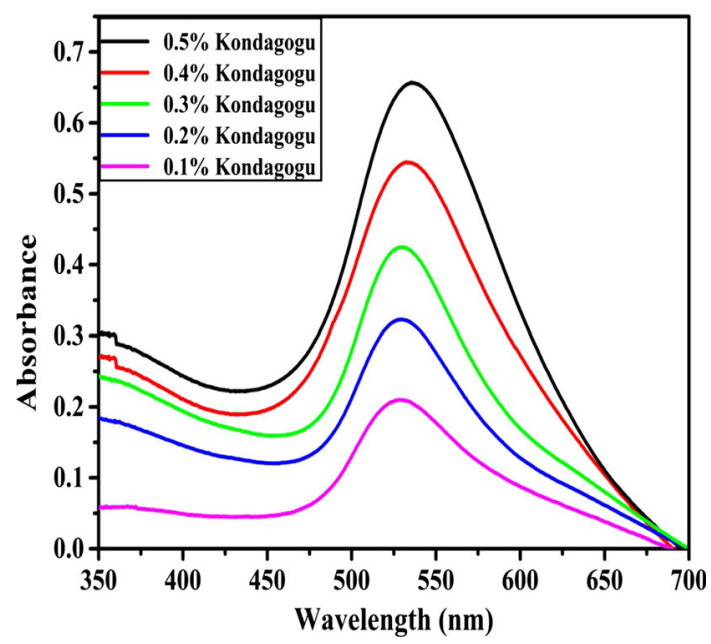

Fig. 1 The UV-Vis absorption spectra of Au nanoparticles synthesized by autoclaving different concentrations of gum kondagogu solution with $1 \mathrm{mM} \mathrm{HAuCl}{ }_{4}$

\section{Results and discussion}

$\mathrm{UV}-\mathrm{V}$ is

UV-Visible spectroscopy is a useful technique to determine the size and stability of nanoparticles [40]. Formation of AuNPs was primarily observed by UV-Visible spectroscopy. The color change is attributed to the SPR phenomenon. The UV-Visible absorption spectra recorded, showed a maximum peak in the wavelength range of around $515-560 \mathrm{~nm}$ as evident from Fig. 1, which is ascribed to the SPR band for AuNPs. The role of gum concentration on the synthesis of nanoparticles was studied by autoclaving different concentrations $(0.1-0.5 \%)$ of gum solutions containing $1 \mathrm{mM}$ of $\mathrm{HAuCl}_{4}$ for $5 \mathrm{~min}$ as shown in Fig. 1. From this it is clear that with an increase in gum concentration there is an enhancement in the formation of nanoparticles [37]. The synthesis of nanoparticles was also examined by changing the concentration of $\mathrm{HAuCl}_{4}$ and the reaction was studied with $0.5 \%$ gum (Fig. 2). It reveals that the amount of nanoparticles synthesized, increases with the increase in concentration of $\mathrm{HAuCl}_{4}$.

\section{FTIR}

Figure $3 \mathrm{a}$ and $\mathrm{b}$ indicate the FTIR spectra of pure gum kondagogu and gum kondagogu capped AuNPs, respectively. The major stretching frequencies in the spectrum of gum kondagogu are observed at 3418, 2943, 1722, 1606, 1422, 1367, 1252, 1149 and $1067 \mathrm{~cm}^{-1}$ [curve (a) of Fig. 3], while the gum kondagogu capped AuNPs showed characteristic stretching frequencies at 3445, 2939, 1723, $1598,1432,1368,1254,1140,1031 \mathrm{~cm}^{-1}$ [curve (b) of 


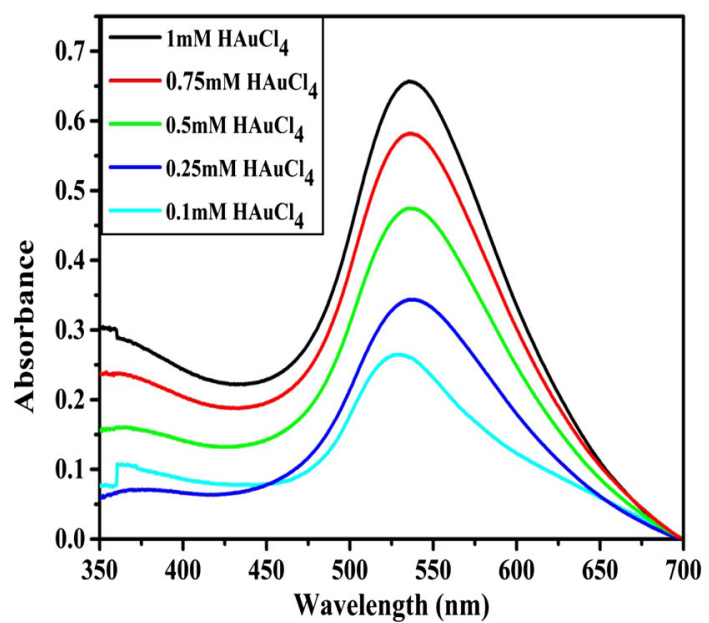

Fig. 2 The UV-Vis absorption spectra of Au nanoparticles synthesized by autoclaving different concentrations of $\mathrm{HAuCl}_{4}$ with $0.5 \%$ gum kondagogu solution

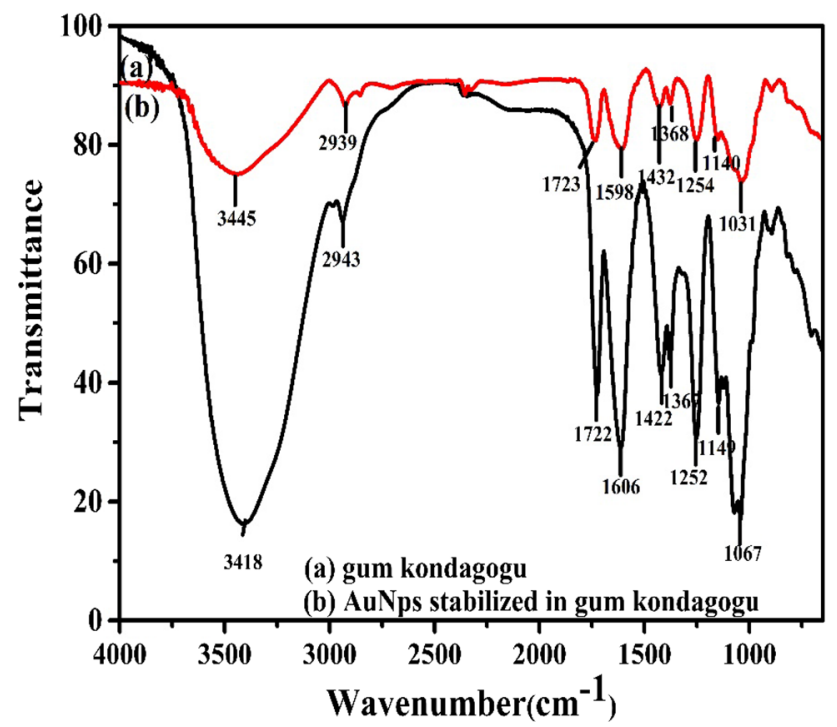

Fig. 3 FTIR spectra of a gum kondagogu, b AuNPs stabilized in gum kondagogu

Fig. 3]. The bands observed at $3445 \mathrm{~cm}^{-1}$ suggest the $-\mathrm{OH}$ group, at $2943 \mathrm{~cm}^{-1}$ the asymmetric $\mathrm{C}-\mathrm{H}$ stretch, at $1722 \mathrm{~cm}^{-1}$ the carbonyl stretching vibration, at $1606 \mathrm{~cm}^{-1}$ the asymmetric stretch of carboxylate, at $1367 \mathrm{~cm}^{-1}$ symmetrical stretch of carboxylate, at $1252 \mathrm{~cm}^{-1}$ the presence of acetyl group, at 1149 and $1067 \mathrm{~cm}^{-1}$ the $\mathrm{C}-\mathrm{O}$ stretching vibration of ether and alcohol groups. A shift in the peaks of the FTIR spectrum of gum kondagogu capped AuNPs was observed from 3418 to $3445 \mathrm{~cm}^{-1}, 1606$ to $1598 \mathrm{~cm}^{-1}$ and the remaining peaks are unchanged suggesting the binding of AuNPs with hydroxyl and carboxylate groups. Based on the band shift in the hydroxyl and carboxyl group, it can be inferred that both hydroxyl

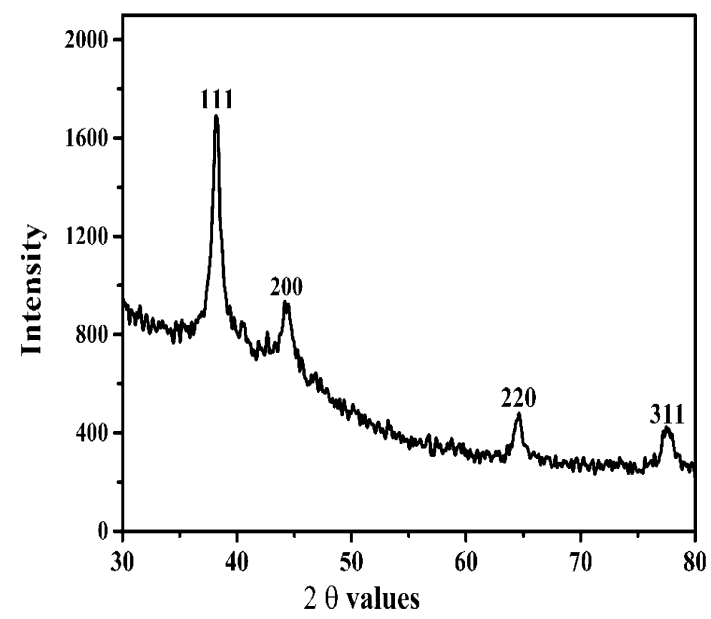

Fig. 4 XRD pattern of gold nanoparticles stabilized in gum kondagogu. Conditions: $0.5 \%$ (w/v) of gum solution, $1 \mathrm{mM}$ of $\mathrm{HAuCl}_{4}$ and autoclaved for $15 \mathrm{~min}$ at $15 \mathrm{psi}$

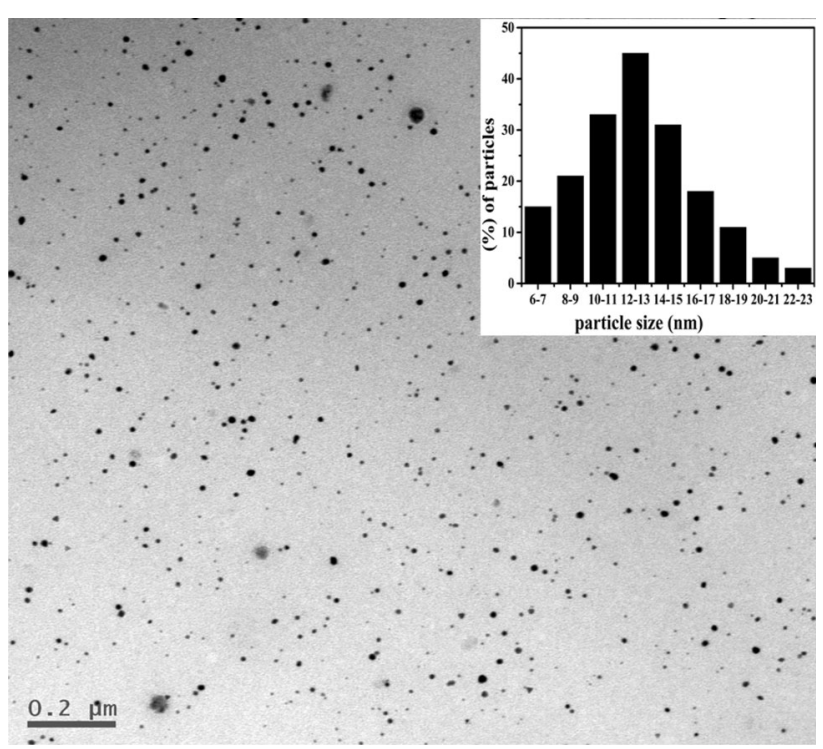

Fig. 5 TEM image of gold nanoparticles synthesized with $1 \%(\mathrm{w} / \mathrm{v})$ gum kondagogu and $1 \mathrm{mM} \mathrm{HAuCl}_{4}$, autoclaved for $15 \mathrm{~min}$ at $15 \mathrm{psi}$ and histogram showing the particle size distribution

and carbonyl groups of gum are involved in the synthesis and stabilization of AuNPs.

\section{XRD}

The XRD technique was used to determine and confirm the crystal structure of AuNPs. For XRD analysis, the prepared sample was lyophilized and the precipitate obtained was kept under vacuum and used for the analysis. The intense diffraction peaks observed at $38.10^{\circ}, 44.16^{\circ}, 64.37^{\circ}$, and $77.51^{\circ}$ were indexed as (111), (200), (220), and (311), 
respectively, as evident from the (Fig. 4). These are the Bragg's reflections of FCC (face centered cubic) structure of crystalline metallic gold (JCPDS No. 04-0784). This fact reveals that the synthesized AuNPs are of pure crystalline AuNPs. The strongest reflection was obtained from (111) inferring that (111) is the predominant orientation and the prepared AuNPs are crystalline in nature. The absence of any further crystallographic impurities and peak broadening in XRD spectrum indicates the high purity of nanocrystalline AuNPs. The XRD pattern, thus, clearly shows that the synthesized AuNPs were essentially crystalline. Crystallite size of AuNPs was calculated using the Scherer's formula from the XRD pattern and was found to be around $13.1 \mathrm{~nm}$. The observations from the XRD analysis are in good agreement with the TEM analysis $(12 \pm 2 \mathrm{~nm})$.

\section{TEM}

The size and shape of the synthesized AuNPs were confirmed by TEM analysis. Figure 5 shows that AuNPs synthesized, were mainly spherical along with a few irregular shapes. It is obvious that the resultant AuNPs were discrete completely, revealing that the gum could protect $\mathrm{Au}$ nanoparticles from aggregation effectively. Histogram constructed by considering 155 nanoparticles suggests that the average size distribution is $12 \pm 2 \mathrm{~nm}$.

Stability of gold nanoparticles against $\mathrm{pH}$ and $\mathrm{NaCl}$

The synthesized, gum-kondagogu-stabilized AuNPs were studied for their stability under different $\mathrm{pH}$ and electrolytic conditions. The effect of $\mathrm{pH}$ on SPR peak was studied by varying $\mathrm{pH} 2-12$ (Fig. 6a). Variation of $\mathrm{pH}$ did not affect the stability of gum-kondagogu-stabilized AuNPs; there is no shift in the SPR peak and the peak intensity was decreased due to dilution. In the second scenario the influence of salt effect on the stability of the gold particles is shown in Fig. 6b. The synthesized AuNPs exhibited very high stability on the addition of varying concentrations $(1-5 \mathrm{M})$ of sodium chloride $(\mathrm{NaCl})$. This is attributed to the fact that the resultant AuNPs were found to be quite stable even in the presence of $\mathrm{NaCl}$, owing to the protection of gum kondagogu on the nanoparticle surface.

\section{Catalytic property}

A potential application of metal nanoparticles is the catalysis of certain reactions, which would not otherwise occur. In the present study, we have taken a model reduction reaction of 4-NP to 4-AP by $\mathrm{NaBH}_{4}$. The substrates and products of this reaction are detected by spectroscopic methods and there is no appreciable formation of
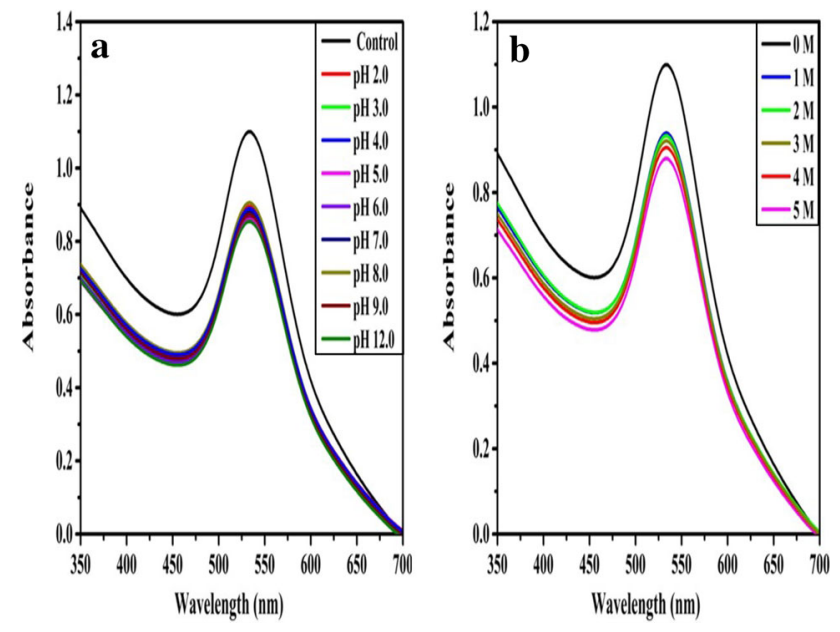

Fig. 6 Effect of $\mathrm{pH}$ (a) and salt concentration (b) on the stability of gum kondagogu capped AuNP

byproducts. The reaction took place after mixing $1.7 \mathrm{~mL}$ of $0.2 \mathrm{mM} 4 \mathrm{NP}$ with $1 \mathrm{~mL}$ of $15 \mathrm{mM} \mathrm{NaBH}_{4}$ in the quartz cell leading to the change of color from light yellow to deep yellow color. 4-NP solution exhibits a strong absorption peak at $317 \mathrm{~nm}$. After the addition of $\mathrm{NaBH}_{4}$, the reaction mixture showed a strong absorption peak at $400 \mathrm{~nm}$ which is due to the formation of $p$-nitrophenolate ion [34]. It is also observed that the $\mathrm{pH}$ of the solution has changed from acidic to highly basic nature.

The reduction of 4-NP to 4-AP using aqueous $\mathrm{NaBH}_{4}$ is thermodynamically favorable $\left(\mathrm{E}^{\circ}\right.$ for $4-\mathrm{NP} / 4-\mathrm{AP}=$ $-0.76 \mathrm{~V}$ and $\mathrm{H}_{3} \mathrm{BO}_{3} / \mathrm{BH}_{4}^{-}=-1.33 \mathrm{~V}$ vs. NHE). The presence of the kinetic barrier due to the large potential difference between donor and acceptor molecules decreases the feasibility of this reaction. No reaction was observed without the addition of Au-based catalyst. There was no change of the peak at $400 \mathrm{~nm}$. This indicates that $\mathrm{NaBH}_{4}$ itself was not able to reduce $p$-nitrophenolate ion directly. AuNPs were added to phenolate ion mixture and were placed in UV-Visible spectrophotometer. The reaction was followed for every 1-min time interval in the range of $200-650 \mathrm{~nm}$. A decrease in the intensity of the absorption peak at $400 \mathrm{~nm}$ was observed, while there is a concomitant appearance of a new peak at $298 \mathrm{~nm}$, indicating the formation of reduced product 4-AP (Fig. 7). A small peak at $530 \mathrm{~nm}$ indicate that there was no significant change observed in the SPR of AuNPs during catalytic reduction of 4-NP. This observation suggested no agglomeration and instability of AuNPs during the catalytic reaction, even in the presence of a higher concentration of reducing agent $\mathrm{NaBH}_{4}$. From this, it is inferred that the catalytic activity of AuNPs was a surface reaction phenomenon.

The rate constant of the reaction is of pseudo first order with respect to 4-NP. The concentration of $\mathrm{NaBH}_{4}$ greatly exceeds that of 4-NP and the reaction rate can be assumed 


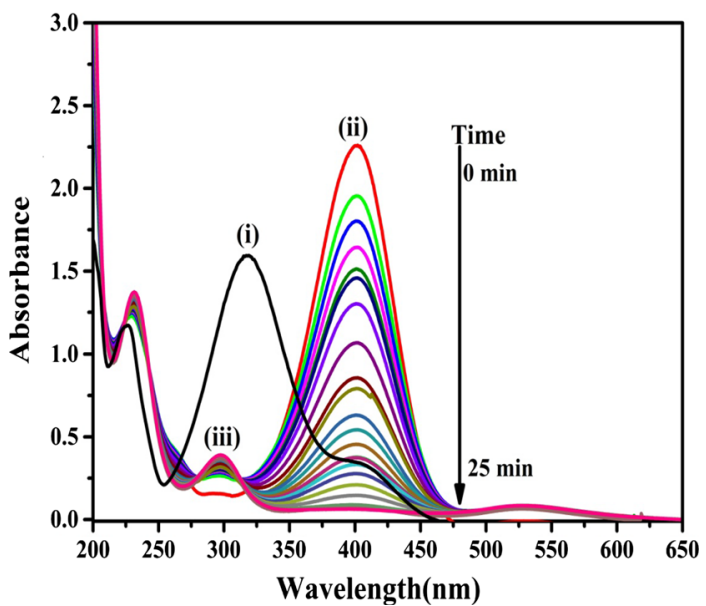

Fig. 7 UV-Visible spectra recorded during the reduction of 4-NP with $\mathrm{NaBH}_{4}$ catalyzed by AuNPs $i$ 4-NP, ii reduction of nitrophenolate ion with time interval of $1 \mathrm{~min}$, iii 4 -AP

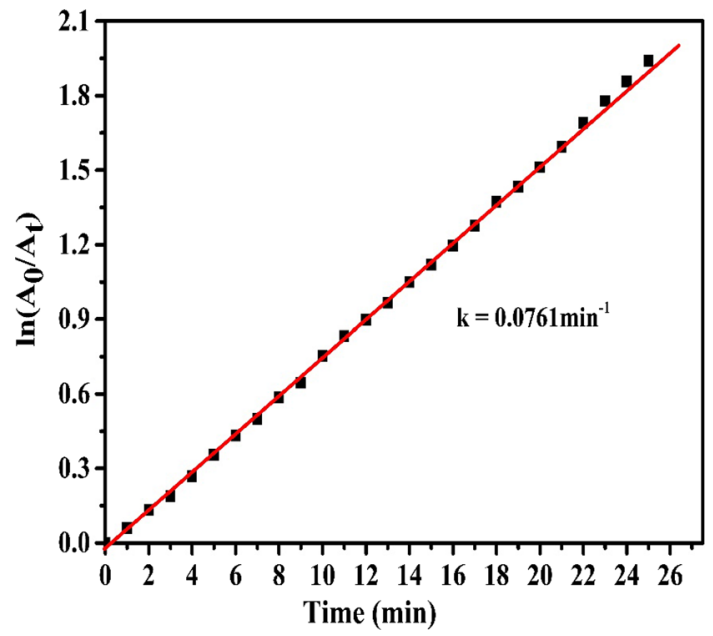

Fig. 8 The plot of $\ln \left(A_{0} / A_{\mathrm{t}}\right)$ versus time for the reduction of nitrophenol to aminophenol

to be independent of $\mathrm{NaBH}_{4}$ concentration. The rate constant $(k)$ was determined from the linear plot of $\ln \left(A_{0} / A_{\mathrm{t}}\right)$ versus reduction time in minutes (Fig. 8). The rate of reduction was influenced by parameters such as concentration of catalyst and temperature. The concentration of $\mathrm{BH}_{4}^{-}$ remains constant during the reaction as the concentration of $\mathrm{NaBH}_{4}$ is very much higher than that of 4-NP.

The effect of the concentration of synthesized AuNPs on the reduction of 4-NP to 4-AP was studied using AuNPs from 50 to $150 \mu \mathrm{L}$ keeping the other parameters such as concentration of $\mathrm{NaBH}_{4}$, temperature and concentration of 4-NP constant. The rate constants for $50,100,150 \mu \mathrm{L}$ catalyst are $0.038,0.0516,0.0761 \mathrm{~min}^{-1}$, respectively. The rate constant values are plotted against varying amount of catalyst as shown in the Fig. 9. As anticipated, the rate constant was enhanced with an increase in the amount of

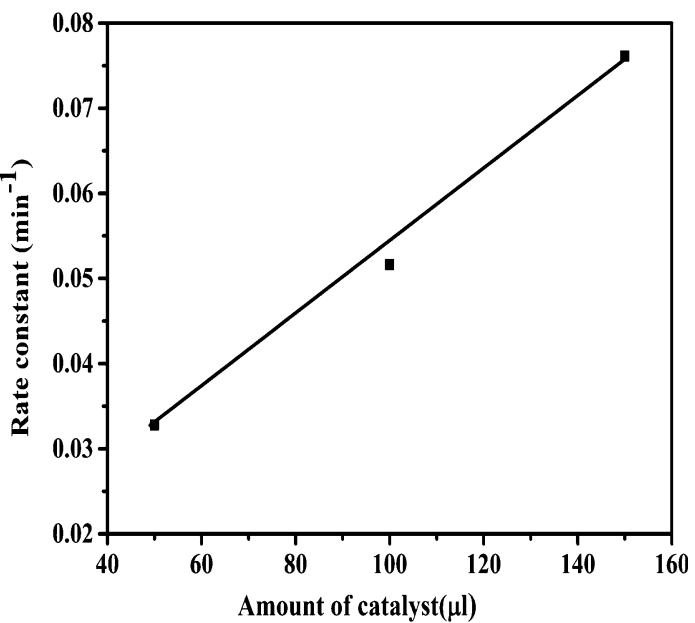

Fig. 9 Plot of rate constant $(k)$ versus amount of AuNPs for the reduction reaction of 4-NP by $\mathrm{NaBH}_{4}$ in the presence of AuNPs as catalyst. Conditions: $[4-\mathrm{NP}]=0.2 \mathrm{mM} ;\left[\mathrm{NaBH}_{4}\right]=15 \mathrm{mM}$; different amounts of gold nanoparticles obtained from $1 \mathrm{mM}$ of chloroauric acid and $0.5 \%$ of gum kondagogu

AuNPs and is evident from Fig. 9. This is due to an increase in the number of reaction sites.

The temperature dependent studies were carried out from 30 to $65{ }^{\circ} \mathrm{C}$ for the reduction of 4-NP to 4-AP. The activation energy $\left(E_{\mathrm{a}}\right)$ of the reaction was obtained from the Arrhenius equation $k=A_{\mathrm{e}}^{-E_{\mathrm{a}} /(R T)}$, where $k$ is rate constant of the reaction at temperature $T$ (in Kelvin), $A$ is a constant and $R$ is the universal gas constant. The catalytic reduction of 4-NP was studied at five different temperatures $(30,35$, 45, 55 and $65{ }^{\circ} \mathrm{C}$ ) using green synthesized AuNPs as catalyst. The rate constant of the reaction was calculated for the five different temperatures. A plot of $\ln k$ versus $1 / T$, shown in Fig. 10, is a linear curve for 4-NP reduction using AuNPs. The Table 1 shows the dependence of the rate constant on temperature. It was observed that the increase in temperature helps the rate of reaction to increase. The activation energy was calculated from the slope $\left(-E_{\mathrm{a}} / R\right)$ of the straight line and was found to be $30.12 \pm 1.34 \mathrm{~kJ} / \mathrm{mol}$.

\section{Antibacterial activity}

AuNPs are proved to be highly potent towards antimicrobial activity. The antimicrobial efficiency of AuNPs increases because of their larger total surface area per unit volume [41]. This enhances its biological activity by increasing the contact area of a metal with a microorganism. The antimicrobial activity of green synthesized AuNPs was carried out by disc diffusion method against different pathogenic bacteria of Gram-negative strains of bacteria (E. coli) and Gram-positive strains of bacteria (B. subtilis). The diameter of inhibition values for the above-mentioned samples was recorded. The bacterial inhibition zones of 


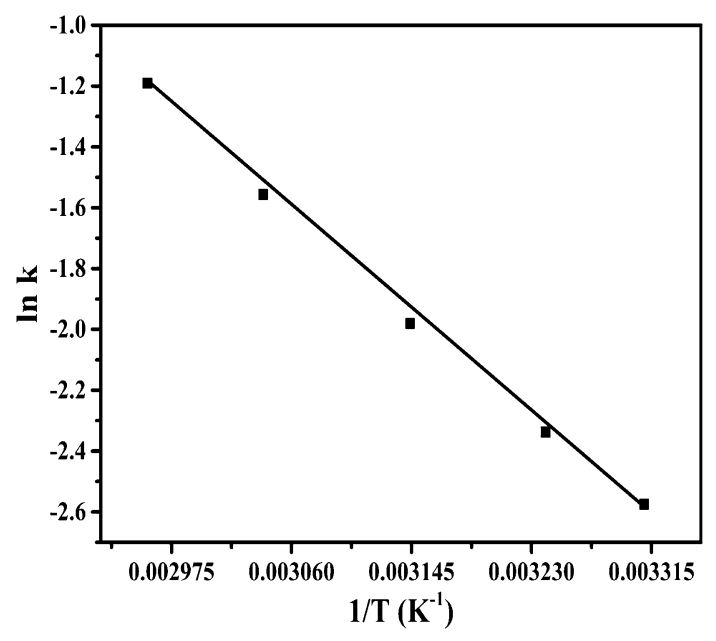

Fig. 10 Plot of rate constant against $1 / T$ for the reduction of 4-NP by $\mathrm{NaBH}_{4}$ in the presence of AuNPs as catalyst at different temperatures. Conditions: $[4-\mathrm{NP}]=0.2 \mathrm{mM}$; $\left[\mathrm{NaBH}_{4}\right]=15 \mathrm{mM} ; 100 \mu \mathrm{L}$ solution of gold nanoparticles obtained from $1 \mathrm{mM}$ of chloroauric acid and $0.5 \%$ of gum kondagogu

Table 1 Rate constants at different temperatures for AuNPs-catalyzed reduction of 4-NP by $\mathrm{NaBH}_{4}$

\begin{tabular}{ll}
\hline Temperature $(K)$ & Rate constant $\left(\mathrm{min}^{-1}\right)$ \\
\hline 303 & $0.0761 \pm 0.01$ \\
308 & $0.0984 \pm 0.013$ \\
318 & $0.1343 \pm 0.014$ \\
328 & $0.2107 \pm 0.018$ \\
338 & $0.3358 \pm 0.012$ \\
\hline
\end{tabular}

Bacillus subtilis and Escherichia coli were observed around the disc, as shown in Fig. 11, while no zone of inhibition was observed for the gum alone. These results revealed that AuNPs synthesized from gum kondagogu demonstrated effective antibacterial activity in Gramnegative than in Gram-positive bacteria. The present results agree well with the work of other researchers [42]. It can be suggested that Gram-negative strains of bacteria $(E$. coli) with thin cell wall is more susceptible to cell wall damage compared to Gram-positive strain bacteria (B. subtilis) with a thick cell wall. The mechanism of the antimicrobial effect of AuNPs is still not well understood. Based on this observation, it can however be concluded that the synthesized AuNPs showed significant antibacterial action on both the Gram classes of bacteria.

\section{Conclusion}

Gum kondagogu is an efficient source for the synthesis of AuNPs. The gum kondagogu acts both as a reductant and as a stabilizer. The synthesized AuNPs were characterized

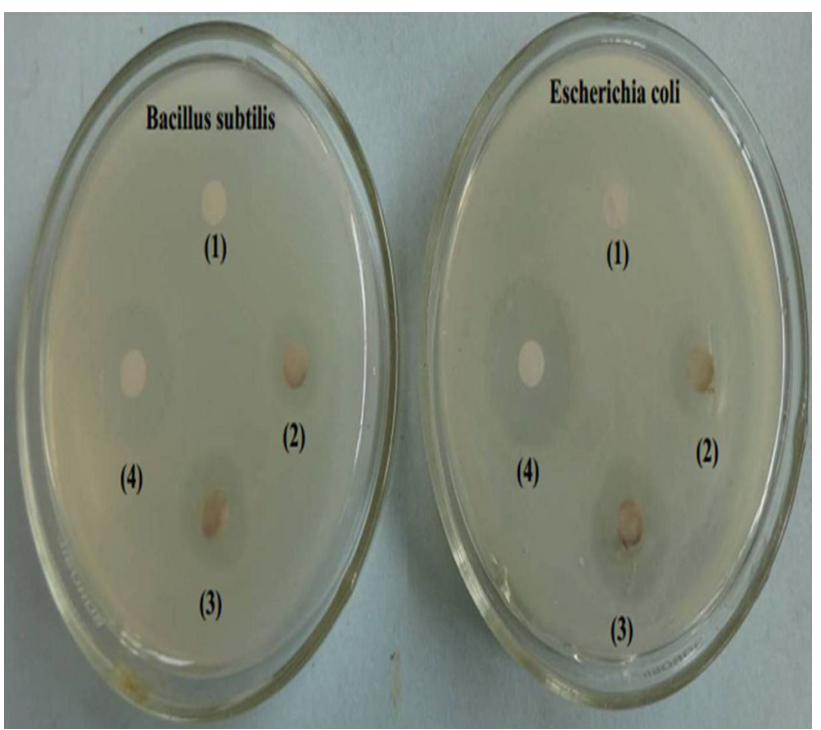

Fig. 11 Antibacterial activity of AuNPs against B. subtilis and E. coli after $24 \mathrm{~h}$ of incubation. $1 \mu \mathrm{L}$ of pure gum kondagogu solution, 2 $5 \mu \mathrm{L}$ of AuNPs, $310 \mu \mathrm{L}$ of AuNPs, $45 \mu \mathrm{L}$ of ampicillin. AuNPs obtained from $1 \mathrm{mM}$ of chloroauric acid and $0.5 \%$ of gum kondagogu

by various techniques. The XRD pattern showed that the synthesized AuNPs were essentially crystalline. It is found that both hydroxyl and carbonyl groups of gum kondagogu are involved in the synthesis and stabilization of AuNPs. The morphology by TEM showed that the synthesized AuNPs were spherical in shape and crystalline in nature with the average size distribution of $12 \pm 2 \mathrm{~nm}$. The catalytic activity of green synthesized AuNPs was examined by the hydrogenation of 4-NP reaction. The detailed kinetic aspects for catalytic hydrogenation were evaluated by changing the process parameters. It is as well observed that with an increase in AuNPs concentration and temperature, the total reaction time decreased and the rate of the reaction has increased. The synthesized AuNPs showed significant antibacterial action on both the gram classes of bacteria (E. coli and B. subtilis).

\section{Methods}

Materials

The starting materials for the synthesis of AuNPs were gum kondagogu (obtained from Girijan Co-operative Corporation Limited, Hyderabad), tetrachloroauric(III) acid trihydrate- $\mathrm{HAuCl}_{4} 3 \mathrm{H}_{2} \mathrm{O}$ (99.9\%, Aldrich), sodium borohydride- $\mathrm{NaBH}_{4}$ (98\%, S-D Fine Chemicals), nitric acid- $\mathrm{HNO}_{3}$ (S-D Fine Chemicals), hydrochloric acid$\mathrm{HCl}$ (S-D Fine Chemicals) and $p$-nitrophenol (S-D Fine chemicals). 
Synthesis of AuNPs

All the solutions were prepared in Milli-Q water. $0.5 \%$ (w/ v) of homogeneous gum stock solution was prepared by adding a calculated quantity of gum kondagogu powder into the reagent bottle containing milli-q water and stirring the same for $1 \mathrm{~h}$ at room temperature. $1 \mathrm{~mL}$ of $1 \mathrm{mM}$ chloroauric acid solution and $3 \mathrm{~mL}$ of gum kondagogu solution were mixed in a boiling tube. This mixture was kept in an autoclave at 15 psi pressure and $120{ }^{\circ} \mathrm{C}$ for $10 \mathrm{~min}$. The resulting solution was red colored, indicating the formation of AuNPs. The solution of synthesized gold nanoparticles was centrifuged at high speed. The pellet and supernatant liquid were separated. The pellet was again dispersed in double-distilled water.

\section{Characterization}

UV-Vis

In order to confirm the formation of AuNPs, the UVVisible absorption spectra of the prepared colloidal solution was recorded using a UV-Vis-NIR spectrophotometer (UV-3600, Shimadzu) having a scanning range of 200-700 nm against blank autoclaved gum.

\section{FTIR}

FTIR analysis was carried out in order to determine the possible functional groups of gum kondagogu, which helps in the reduction and stabilization agent of synthesized nanoparticles. The colloidal solution of AuNPs was first lyophilized and the sample was used for FTIR analysis in the form of a thin transparent pellet with $\mathrm{KBr}$. A pure $\mathrm{KBr}$ pellet was used as a background and this was subtracted from the FTIR spectra of the gum kondagogu and AuNPs sample. FTIR spectra were recorded with an instrument IR Affinity1 (Shimadzu) in the scanning range of $650-4000 \mathrm{~cm}^{-1}$.

\section{XRD}

The crystallinity of the AuNPs was studied by XRD (Rigaku, Miniflex) method with Cuk $\alpha$ radiation.

\section{TEM}

The morphology and size of synthesized AuNPs was examined by TEM. The sample gird for TEM measurement was prepared by placing a drop of aqueous AuNPs dispersion on the carbon-coated copper grid and subsequently evaporating the water naturally overnight at ambient conditions. The measurements were done on JEOL 2000 FX-II TEM.

Catalytic reduction of $p$-nitrophenol

As a sample reaction, the reduction of $p$-nitrophenol to $p$ aminophenol by sodium borohydride has been selected. The reduction took place in aqueous solution in a standard quartz cell with $1 \mathrm{~cm}$ path length. In the reaction process, $1.7 \mathrm{~mL}$ of $0.2 \mathrm{mM} p$-nitrophenol was mixed with $1.0 \mathrm{~mL}$ of $0.015 \mathrm{M} \mathrm{NaBH}_{4}$ in the cell for UVvisible measurements. Immediately, the color changed from light yellow to deep yellow. Varying concentrations of $(50-150 \mu \mathrm{L})$ of AuNPs solution was added to the above mixture. The UV-visible absorption spectra were recorded with a time interval of $1 \mathrm{~min}$, in a scanning range of $200-650 \mathrm{~nm}$.

Antibacterial property of AuNPs

Antibacterial properties of the synthesized AuNPs were carried out using the disc diffusion method. Gram-positive and Gram-negative bacteria, Bacillus subtilis and Escherichia coli, respectively, were used as model test strains. Luria-Bertani (LB) agar medium was prepared and transferred to sterilized petri dishes. The medium was allowed to solidify and then the petri plates were spread with Bacillus subtilis and Escherichia coli separately in a laminar air flow hood. Using micropipette, 5 and $10 \mu \mathrm{L}$ of the AuNPs solution and $5 \mu \mathrm{L}$ of gum kondagogu solutions added to each well on both plates. The discs were air dried in laminar hood and incubated at $37{ }^{\circ} \mathrm{C}$ for $24 \mathrm{~h}$. Then, zone of inhibition of bacteria was measured. The assays were performed in triplicate.

Acknowledgments One of the authors, Bhagavanth Reddy G, gratefully acknowledges CSIR, New Delhi, for providing Senior Research Fellowship. The authors wish to thank Centre for Nanotechnology, University of Hyderabad, for allowing the use of their TEM facility.

Open Access This article is distributed under the terms of the Creative Commons Attribution License which permits any use, distribution, and reproduction in any medium, provided the original author(s) and the source are credited.

\section{References}

1. Murray, R.W.: Nanoelectrochemistry: metal nanoparticles, nanoelectrodes, and nanopores. Chem. Rev. 108, 2688-2720 (2008)

2. Sardar, R., Funston, A.M., Mulvaney, P., Murray, R.W.: Gold nanoparticles: past, present, and future. Langmuir $\mathbf{2 5}$, 13840-13851 (2009)

3. Paramaconi, R., Daniela, P., David, J.F., Marc, T.M.K.: New insights into the catalytic activity of gold nanoparticles for $\mathrm{CO}$ 
oxidation in electrochemical media. J. Catal. 311, 182-189 (2014)

4. Lin, C.Y., Yu, C.J., Lin, Y.H., Tseng, W.L.: Colorimetric sensing of silver(I) and mercury(II) ions based on an assembly of Tween 20-stabilized gold nanoparticles. Anal. Chem. 82, 6830-6837 (2010)

5. Wang, W., Tao, N.: Detection, counting, and imaging of single nanoparticles. Anal. Chem. 86, 2-14 (2014)

6. Lu, F., Doane, T.L., Zhu, J., Burda, C.: Gold nanoparticles for diagnostic sensing and therapy. Inorg. Chim. Acta 393, 142-153 (2012)

7. Yang, Y., Matsubara, S., Nogami, M., Shi, J., Huang, W.: Onedimensional self-assembly of gold nanoparticles for tunable surface plasmon resonance properties. Nanotechnology 17, 2821-2827 (2006)

8. Sharma, H., Agarwal, D.C., Shukla, K., Avasthi, D.K., Vankar, V.D.: Surface-enhanced Raman scattering and fluorescence emission of gold nanoparticle-multiwalled carbon nanotube hybrids. J. Raman Spectrosc. 44, 12-20 (2013)

9. Schmid, G., Simon, U.: Gold nanoparticles: assembly and electrical properties in 1-3 dimensions. Chem. Commun. (2005). doi: $10.1039 / \mathrm{b} 411696 \mathrm{~h}$

10. Gréget, R., Gareth, L.N., Bertrand, V., Philippe, T.: Magnetic properties of gold nanoparticles: a room-temperature quantum effect. ChemPhysChem 13, 3092-3097 (2012)

11. Shalkevich, N., Werner, E., Thomas, B., Bruno, M., Si-Ahmed, L., Poulikakos, D.: On the thermal conductivity of gold nanoparticle colloids. Langmuir 26, 663-670 (2010)

12. Gao, J., Huang, X., Liu, H., Zan, F., Ren, J.: Colloidal stability of gold nanoparticles modified with thiol compounds: bioconjugation and application in cancer cell imaging. Langmuir 28, 4464-4471 (2012)

13. Nagajyothi, PC, Lee, KD, Sreekanth, TVM.: Biogenic synthesis of gold nanoparticles (quasi-spherical, triangle, and hexagonal) using Lonicera japonica flower extract and its antimicrobial activity. Synth. React. Inorganic, Met. Nano-Metal Chem. 44, 1011-1018 (2014)

14. Aghdam, A.G., Vossoughi, M., Almzadeh, I., Zeinali, M.: Bioconjugation of interferon-alpha molecules to lysine-capped gold nanoparticles for further drug delivery applications. J. Dispers. Sci. Technol. 29, 1062-1065 (2008)

15. Delong, R.K., Reynolds, C.M., Malcolm, Y., Schaeffer, A., Severs, T., Wanekaya, A.: Functionalized gold nanoparticles for the binding, stabilization, and delivery of therapeutic DNA, RNA, and other biological macromolecules. Nanotechnol. Sci. Appl. 3, 53-63 (2010)

16. Eustis, S., Hsu, H., El-sayed, M.A.: Gold nanoparticle formation from photochemical reduction of Au3 + by continuous excitation in colloidal solutions. A proposed molecular mechanism. J. Phys. Chem. B 109, 4811-4815 (2005)

17. Ojea-jime, I., Romero, F.M., Bastu, N.G., Puntes, V.: Small gold nanoparticles synthesized with sodium citrate and heavy water : insights into the reaction mechanism. J. Phys. Chem. C 114, 1800-1804 (2010)

18. Jagtap, NR, Shelke, VA, Nimase, MS, Jadhav, SM, Shankarwar, SG, Chondhekar, TK.: Electrochemical synthesis of tetra alkyl ammonium salt stabilized gold nanoparticles. Synth. React. Inorganic, Met. Nano-Metal Chem. 42, 1369-1374 (2012)

19. Farkas, J., Christian, P., Urrea, J.A., Roos, N., Hassellövd, M., Tollefsen, K.E.: Effects of silver and gold nanoparticles on rainbow trout (Oncorhynchus mykiss) hepatocytes. Aquat. Toxicol. 96, 44-52 (2010)

20. Sharma, N.C., Torresdey, J.L.: Synthesis of plant-mediated gold nanoparticles and catalytic role of biomatrix-embedded nanomaterials. Environ. Sci. Technol. 41, 5137-5142 (2007)

21. Kumar, K.P., Paul, W., Sharma, C.P.: Green synthesis of gold nanoparticles with Zingiber officinale extract: characterization and blood compatibility. Process Biochem. 46, 2007-2013 (2011)

22. Malarkodi, C., Rajeshkumar, S., Vanaja, M., Paulkumar, K., Gnanajobitha, G., Annadurai, G.: Eco-friendly synthesis and characterization of gold nanoparticles using Klebsiella pneumoniae. J. Nanostruct. Chem. 3, 1-7 (2013)

23. Gopinath, K., Gowri, S., Karthika, V.V., Arumugam, A.: Green synthesis of gold nanoparticles from fruit extract of Terminalia arjuna, for the enhanced seed germination activity of Gloriosa superba. J. Nanostruct. Chem. 4, 1-11 (2014)

24. Dauthal, P., Mukhopadhyay, M.: Prunus domestica fruit extractmediated synthesis of gold nanoparticles and its catalytic activity for 4-nitrophenol reduction. Ind. Eng. Chem. Res. 51, 13014-13020 (2012)

25. Dhar, S., Maheswara, R.E., Prabhune, A., Pokharkar, V., Anjali, S., Prasad, B.L.V.: Cytotoxicity of sophorolipid-gellan gum-gold nanoparticle conjugates and their doxorubicin loaded derivatives towards human glioma and human glioma stem cell lines. Nanoscale 3, 575-580 (2011)

26. Wu, Y., Zuo, F., Lin, Y., Zhou, Y., Zhaohui, Z., Ding, X.: Green and facile synthesis of gold nanoparticles stabilized by chitosan. J. Macromol. Sci. Part A 51, 441-446 (2014)

27. Maity, S, Kumar Sen, I, Sirajul, IS.: Green synthesis of gold nanoparticles using gum polysaccharide of Cochlospermum religiosum (katira gum) and study of catalytic activity. Phys. E. 45, 130-134 (2012)

28. Janaki, B., Sashidhar, R.B.: Sub-chronic (90-day) toxicity study in rats fed gum kondagogu (Cochlospermum gossypium). Food Chem. Toxicol. 38, 523-534 (2000)

29. Vinod, V.T.P., Sashidhar, R.B.: Surface morphology, chemical and structural assignment of gum kondagogu (Cochlospermum gossypium DC.): an exudate tree gum of India. Indian J. Nat. Prod. Resour. 1, 181-192 (2010)

30. Rastogi, L., Sashidhar, R.B., Karunasagar, D., Arunachalam, J.: Gum kondagogu reduced/stabilized silver nanoparticles as direct colorimetric sensor for the sensitive detection of $\mathrm{Hg}^{+2}$ in aqueous system. Talanta 118, 111-117 (2014)

31. Carregal, R.S., Perez, J.J., Herv, P., Liz-Marzan, L.M., Mulvaney, P.: Colloidal gold-catalyzed reduction of ferrocyanate(III) by borohydride ions: a model system for redox catalysis. Langmuir 26, 1271-1277 (2010)

32. Stratakis, M., Garcia, H.: Catalysis by supported gold nanoparticles: beyond aerobic oxidative processes. Chem. Rev. 112, 4469-4506 (2012)

33. El-Sheikh, S.M., Ismail, A.A., Al-Sharab, J.F.: Catalytic reduction of $p$-nitrophenol over precious metals/highly ordered mesoporous silica. New J. Chem. 37, 2399-2407 (2013)

34. Banik, R.M., Prakash, R., Upadhyay, S.N.: Microbial biosensor based on whole cell of Pseudomonas sp. for online measurement of $p$-Nitrophenol. Sens. Actuators B Chem. 131, 295-300 (2008)

35. Ju, K.S., Parales, R.E.: Nitroaromatic compounds, from synthesis to biodegradation. Microbiol. Mol. Biol. Rev. 74, 250-272 (2010)

36. Barad, J., Chakraborty, M.: Reduction of 4-nitrophenol and 4-nitrobenzo 15 crown with colloidal platinum nanoparticles synthesized by microemulsion technique. Part. Sci. Technol. 32, 164-170 (2014)

37. Venkatesham, M., Ayodhya, D., Madhusudhan, A., Veerabhadram, G., Girija, M.K.: A novel green synthesis of silver nanoparticles using gum karaya: characterization, antimicrobial and catalytic activity studies. J. Clust. Sci. 25, 409-422 (2013)

38. Catalina, M.J., Eric, M.V.: A review of the antibacterial effects of silver nanomaterials and potential implications for human health and the environment. J. Nanopart. Res. 12, 1531-1551 (2010)

39. Ameer, A., Arham, S.A., Oves, M., Khan, S.M.: Antimicrobial activity of metal oxide nanoparticles against Gram-positive and Gram-negative bacteria: a comparative study. Int J Nanomed. 7, 6003-6009 (2012) 
40. Martínez, J.A., Chequer, N.L., González, J., Cordova, T.: Alternative metodology for gold nanoparticles diameter characterization using PCA technique and UV-VIS spectrophotometry. Nanosci. Nanotechnol. (2013). doi:10.5923/j.nn.20120206.06

41. Lokina, S., Narayanan, V.: Antimicrobial and anticancer activity of gold nanoparticles synthesized from grapes fruit extract. Chem. Sci. Trans. (2013). doi:10.7598/cst2013.22
42. El-Batal, A.I., Hashem, A.A., Abdelbaky, N.M.: Gamma radiation mediated green synthesis of gold nanoparticles using fermented soybean-garlic aqueous extract and their antimicrobial activity. Springerplus 2, 1-10 (2013) 